\title{
Diagnóstico de la fertilidad de los suelos de cuatro municipios de Cundinamarca (Colombia) para la producción de plátano
}

\section{Diagnostics of soil fertility in four municipalities of Cundinamarca (Colombia) employed for cooking banana production}

\author{
Sebastián David García Guzmán; Luis Gabriel Bautista-Montealegre²; Martha Marina Bolaños-Benavides ${ }^{3 *}$
}

1'Ingeniero Agrónomo. Universidad Nacional de Colombia, sede Bogotá, e-mail: sdgarciag@unal.edu.co, Dhttps://orcid.org/0000-0001-8739-3334

I.A., MSc, Fitopatología. Universidad Nacional de Colombia, sede Bogotá, e-mail: Igbautistam@unal.edu.co, Dhttps://orcid.org/0000-0001-9305-5630

${ }^{3}$ Investigador, Ph.D.. Corporación Colombiana de Investigación Agropecuaria - AGROSAVIA, C.I. Tibaitatá, Mosquera, e-mail: mmbolanos@agrosavia.co, (D)https://orcid.org/0000-0003-4593-5523.

*autor para correspondencia: mmbolanos@agrosavia.co

Cómo citar: García Guzmán, S.D.; Bautista-Montealegre, L.G.; Bolaños-Benavides, M.M. 2019. Diagnóstico de la fertilidad de los suelos de cuatro municipios de Cundinamarca (Colombia) para la producción de plátano. Rev. U.D.C.A Act. \& Div. Cient. 22 (1):e1192. https://doi.org/10.31910/rudca.v22.n1.2019.1192

Artículo de acceso abierto publicado por Revista U.D.C.A Actualidad \& Divulgación Científica, bajo una licencia Creative Commons CC BY-NC 4.0

Recibido: Febrero 22 de 2018

Aceptado: Marzo 14 de 2019

\section{RESUMEN}

Para lograr rendimientos óptimos en la producción de plátano, se debe tener en cuenta la disponibilidad de nutrientes en el suelo, para así ajustar planes de fertilización adecuados. Con el fin de contribuir al estudio de la fertilidad de suelos cultivados con plátano en el departamento de Cundinamarca-Colombia, se realizó una caracterización química de suelos, en cuatro de los principales municipios productores. Se visitaron 146 fincas, ubicadas en Viotá, La Vega, La Palma y Chaguaní, donde se tomaron muestras de suelo, para análisis químico. Los resultados obtenidos fueron sometidos a un análisis de frecuencias, correlación lineal de Pearson y componentes principales, realizando, finalmente, una comparación de medias entre los cuatro municipios. Se observaron niveles bajos de P, S y $\mathrm{Mg}$, medios de $\mathrm{K}$, Ca y B, así como altas concentraciones de Zn, que pueden llegar a ser tóxicas para el cultivo de plátano. Por otro lado, las variables se agruparon en cuatro componentes asociados a: la capa de cambio del suelo, la reacción del suelo, la salinidad y la relación entre el P y Zn. Suelos de los municipios de La Vega y La Palma no presentaron variación en la capa de cambio y la reacción del suelo, caso contrario al observado en suelos de Viotá y Chaguaní. Entre suelos de los municipios, se presentaron diferencias significativas para las propiedades materia orgánica, Ca y Mg, así como desbalances catiónicos y $\mathrm{pH}$ fuertemente ácidos.

Palabras clave: enmiendas del suelo; Musa spp. AAB group; acidez intercambiable; fitotoxicidad.

\section{ABSTRACT}

To achieve optimum yields in plantain production, appropriate fertilization plans must be adjusted, for which the availability of nutrients in the soil must be taken into account. In order to contribute to the study of the fertility of soils cultivated with cooking banana in the province of Cundinamarca - Colombia, a chemical characterization of soils was carried out in four of the main producing municipalities. 146 farms located in Viotá, La Vega, La Palma and Chaguaní, were visited, and soil samples were taken for chemical analysis. The results obtained were subjected to a frequency analysis, Pearson's linear correlation and main components; finally making a comparison of 
means between the four municipalities. Low levels of $\mathrm{P}, \mathrm{S}$ and $\mathrm{Mg}$, medium of $\mathrm{K}$, Ca and $\mathrm{B}$ were observed, as well as high concentrations of $\mathrm{Zn}$ that can be toxic for the plantain crop. On the other hand, the variables were grouped into four components associated with: the soil change layer, the soil reaction, the salinity and the relationship between $\mathrm{P}$ and $\mathrm{Zn}$. Soils of the municipalities of La Vega and La Palma did not show variation in the change layer and soil reaction, contrary to that observed in Viotá and Chaguaní soils. Among the soils of the municipalities, there were significant differences for the properties of organic matter, $\mathrm{Ca}$ and $\mathrm{Mg}$ as well as cationic imbalances and strongly acidic $\mathrm{pH}$.

Keywords: soil amendments; Musa spp. AAB group; exchangeable acidity; phytotoxicity.

\section{INTRODUCCIÓN}

El banano y el plátano ocupan el cuarto lugar entre los cultivos más importantes del mundo después de cereales, como el arroz y el trigo (Canto et al. 2015). La producción de plátano es de 31 millones de toneladas, a nivel mundial, siendo Uganda el mayor productor, seguido por Camerún y Ghana (Sinagap, 2015). En 2015, Colombia ocupó el segundo lugar en la producción de plátano $\mathrm{AAB}$, con 2.657.910 ton (Lescot, 2015). En este contexto, en el departamento de Cundinamarca, se encuentran sembradas $12.173 \mathrm{ha}$, con un rendimiento de $7 \mathrm{t} \mathrm{ha}^{-1}$ (DANE, 2015). Uno de los componentes de la producción de frutales y, en general, de cualquier cultivo es la fertilización; para ello, se requiere información inicial de la fertilidad de los suelos, con el fin de generar recomendaciones de aplicación de nutrientes, de acuerdo con los requerimientos de las plantas y su fenología.

Para lograr rendimientos óptimos, se debe tener en cuenta que la producción agrícola es resultado de la interacción de factores bióticos y abióticos, siendo la fertilización uno de los más determinantes. Las plantas requieren de nutrientes esenciales para completar su ciclo biológico. En este sentido, resulta necesario asegurar su suficiencia al momento de establecer las plantaciones, ya que un inadecuado manejo de la nutrición puede generar pérdidas de rendimientos (Zoppolo \& Fasiolo, 2016). A pesar de la importancia de la práctica de fertilización, actualmente, muchos productores realizan la aplicación de fertilizantes sin tener en cuenta ningún tipo de análisis, lo cual, genera pérdidas económicas y efectos negativos en el ambiente, por el uso innecesario o excesivo de éstos. Adicionalmente, se deben considerar las interacciones que los organismos puedan generar, ya que estas son indispensables en la disponibilidad de los nutrimentos para la planta.

Debido a la necesidad de fortalecer la agricultura de precisión, se han desarrollado herramientas, que permiten el diagnóstico del estado nutricional de los cultivos por sitio específico, como lo son los análisis de suelos, permitiendo analizar características del sistema suelo-planta, para así lograr un manejo racional de la fertilidad (Castañeda et al. 2014). Para contribuir al estudio de la fertilidad de suelos cultivados con plátano en el departamento de Cundinamarca, se realizó una caracterización química de suelo, en cuatro municipios, con el fin de brindar información base, para la generación de recomendaciones de fertilización, en el sistema productivo de plátano.

\section{MATERIALES Y MÉTODOS}

Ubicación del estudio. Se tomaron muestras de suelo entre enero y marzo de 2017, en fincas cultivadas con plátano dominico-hartón, en los municipios de Cundinamarca, priorizados por su participación en la producción del departamento. Las fincas, se caracterizan por la siembra de plátano, a distancias de 2.5 a $3 \mathrm{~m}$ entre plantas y entre hileras, en asocio con cultivos de café. Se seleccionaron 53, en el municipio de Viotá; 43, en La Vega; 21, en La Palma y 29, en Chaguaní, para un total de 146. En la mayoría de las fincas analizadas, las diferentes prácticas de manejo de la fertilidad, se realizan sin ningún tipo de análisis o recomendación y, además, están asociadas al café y, por lo tanto, el plátano se puede ver afectado negativamente, ya que sus niveles de extracción de nutrientes son diferentes; sumado a esto, los agricultores poseen malas prácticas de cultivo (aplicación de Cal dolomita antes de iniciar cada ciclo, erradicación de malezas con $\mathrm{Cl}$, entre otras), como consecuencia a la carencia de asistencia técnica. En los lotes, se realizó un muestreo compuesto obteniendo un kg de suelo, el cual, fue llevado al Laboratorio de Suelos de Agrosavia, C.I. Tibaitatá, para su posterior análisis.

Análisis químico de las muestras de suelo. Mediante los protocolos establecidos por el laboratorio, en el análisis químico de suelo, se determinó pH (potenciómetro; relación suelo-agua 1:2,5), acidez y Al intercambiables ( $\mathrm{KCl} 1 \mathrm{~N}), \mathrm{MO}$ (Walkley-Black Modificado), P (Bray II), S y B (fosfato monocálcio), bases intercambiables (Ca, $\mathrm{Mg}, \mathrm{K}, \mathrm{Na}$, en acetato de amonio $1 \mathrm{M}$ a pH 7), CICE (Capacidad de Intercambio Catiónico Efectiva), elementos menores ( $\mathrm{Fe}, \mathrm{Cu}, \mathrm{Mn}$, Zn; método Olsen modificado) y conductividad eléctrica.

Análisis estadístico. Con el fin de facilitar la interpretación de los resultados, se efectuó análisis descriptivo de las propiedades químicas, mediante análisis de frecuencias de rangos de la concentración de nutrientes, según la clasificación del ICA (1992). Adicionalmente, se llevó a cabo un análisis de varianza con prueba de Tukey $(\mathrm{P} \leq 0,05$ de significancia), para comparar los niveles de fertilidad de los suelos, en los cuatro municipios, así como un análisis de correlación lineal simple de Pearson y una agrupación de variables, mediante un análisis componentes principales (ACP).

\section{RESULTADOS Y DISCUSIÓN}

Niveles de disponibilidad y de correlación entre elementos. Se observó una mayor frecuencia de fincas con suelos extremadamente ácidos, al reportar pH por debajo de 5,5 (ICA, 1992) (Figura 1). Cuando un suelo posee está condición, se presenta disminución considerable en la capacidad de retención de nutrientes, ya que los espacios de intercambio son ocupados por diferentes iones $(\mathrm{Al}+3$, $\mathrm{H}+, \mathrm{NH}^{+}$, entre otros), que aumentan la acidez del suelo, reduciendo la participación de las bases intercambiables (Ca, Mg y K) (Sadeghian, 2016). En este sentido, se recomiendan prácticas de encalado con sales básicas, con el fin de neutralizar la acidez existente. 
Los productos que se utilizan como correctivos y para aumentar el pH del suelo están compuestos por carbonatos, óxidos, hidróxidos y silicatos de $\mathrm{Ca}$ o $\mathrm{Mg}$, siendo la cal dolomita la más utilizada que, a su vez, contribuye a la disminución de las deficiencias de $\mathrm{Mg}$ y los desbalances existentes en las relaciones de bases catiónicas del suelo (Espinosa \& Molina, 1999).
La acidez del suelo en las regiones tropicales está determinada por procesos edafogenéticos, intensos procesos de lixiviación, a causa de los niveles altos de pluviosidad; altos contenidos de Al y Mn; alta capacidad de fijación de $\mathrm{P}$, asociada al predominio de óxidos de $\mathrm{Fe}$ y Al; liberación de $\mathrm{H}^{+}$por las raíces, debido a la absorción de Ca, Mg y K y procesos de descomposición de materia orgánica (MO),

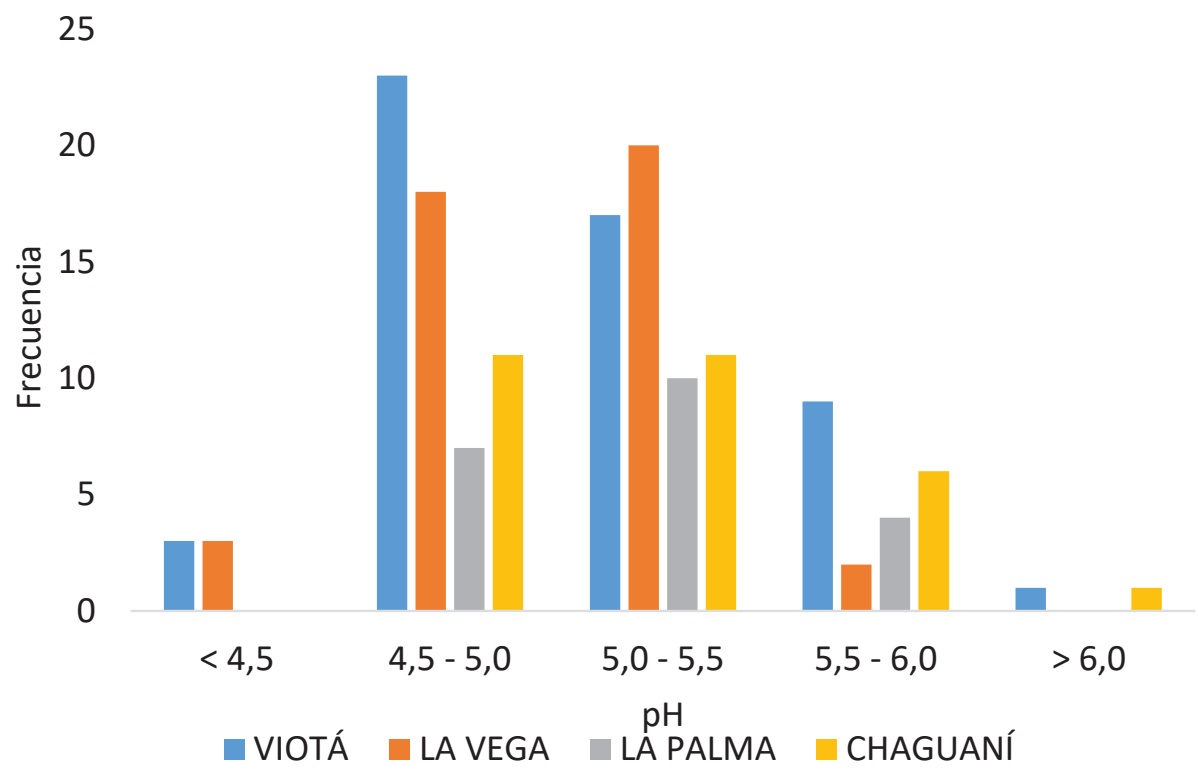

Figura 1. Análisis de frecuencia para el pH del suelo, según una muestra de 146 fincas cultivadas con plátano, de los municipios de Viotá, La Vega, La Palma y Chaguaní, en el departamento de Cundinamarca (Colombia). Los rangos corresponden a los reportados en la Quinta aproximación del ICA (1992).

la cual, tiende a liberar ácidos orgánicos. Además, esta también se puede asociar a prácticas agronómicas mal realizadas sobre las plantaciones (Meléndez \& Molina, 2001). Según los datos recopilados, se obtuvo una relación significativa entre el $\mathrm{pH}$ y la $\mathrm{MO}(\mathrm{r}=0,26$, $\mathrm{P}<0,01)$; aun así, esta fue muy baja. En cuanto a los contenidos de Ca y $\mathrm{Mg}$, se observó una correlación positiva con el $\mathrm{pH}(\mathrm{r}=0,74$, $\mathrm{P}<0,01$ y $\mathrm{r}=0,64, \mathrm{P}<0,01$, respectivamente), que es de esperar, debido a que un suelo con mayores cantidades de $\mathrm{Ca}$ y $\mathrm{Mg}$ tiende a ser más básico (Zapata, 2004).

Por otro lado, los suelos en estudio presentaron correlación negativa entre el $\mathrm{pH}$ y la acidez intercambiable $(\mathrm{Al}+\mathrm{H})$, lo que significa que a menor $\mathrm{pH}$ se incrementará la acidez $(\mathrm{r}=-0,65, \mathrm{P}<0,01)$. En este sentido, al aumentar $1 \mathrm{cmol}^{+} \mathrm{kg}^{-1}$ de $\mathrm{Al}+\mathrm{H}$ el $\mathrm{pH}$ disminuye 0,11 unidades. El contenido promedio de $\mathrm{Al}$ en el suelo fue de $1,68 \mathrm{cmol}^{+}$ $\mathrm{kg}^{-1}$, que corresponde a una saturación de $27,53 \%$, en el complejo de cambio, niveles que pueden llegar a ser tóxicos para el plátano, debido a que, para este cultivo, se recomienda una saturación $<25 \%$, para que el $\mathrm{Al}$ no afecte negativamente el crecimiento y el desarrollo de las plantas (Espinosa \& Molina, 1999). Esta condición, se puede corregir por medio de la adición de $\mathrm{MO}$ al suelo, con el fin de promover la formación de complejos entre ácidos orgánicos y $\mathrm{Al}$ (Kerven et al. 1989; Casierra \& Aguilar, 2007).
Los niveles de $\mathrm{MO}$ en los suelos de la mayoría de las fincas de los cuatro municipios (116) fueron de medios a altos (Figura 2). Éstos, se correlacionaron de manera negativa con los contenidos disponibles de $\mathrm{Fe}(\mathrm{r}=-0,51, \mathrm{P}<0,01)$, lo que significa que, a mayores contenidos de MO, habrá menos Fe en la solución del suelo. Esta condición, se debe a que los óxidos de Fe tienen la facilidad de cambiar de valencia y formar complejos con diferentes materiales, tanto de naturaleza mineral como orgánica. Por lo tanto, la distribución y la disponibilidad de Fe en la solución del suelo, en parte, depende del contenido de materiales orgánicos existentes (Acevedo-Sandoval et al. 2004).

Con relación al $\mathrm{P}$, se presentan niveles bajos $\left(<20 \mathrm{mg} \mathrm{kg}^{-1}\right)$, en la mayoría de las fincas de los cuatro municipios. Esto puede estar relacionado con la presencia de fosfatos de $\mathrm{Fe}$ y Al, compuestos insolubles formados en suelos ácidos, disminuyendo así la disponibilidad del P (Havlin et al. 1999; Acevedo-Sandoval et al. 2004). Además, se presentó una correlación positiva con el $\mathrm{Zn}(\mathrm{r}=0,65$, $\mathrm{P}<0,01)$, debido a que el $\mathrm{Zn}$ posee afinidad con el $\mathrm{P}$, formando fosfatos de $\mathrm{Zn}$; aun así, los niveles de $\mathrm{Zn}$ fueron altos. Acorde con lo anterior, para facilitar la absorción de $\mathrm{P}$, es necesario incluir, en los planes de fertilización, microorganismos que solubilicen las reservas de P (Paredes-Mendoza \& Espinosa-Victoria, 2010); de éstos, se conocen varios géneros de hongos formadores de micorrizas 

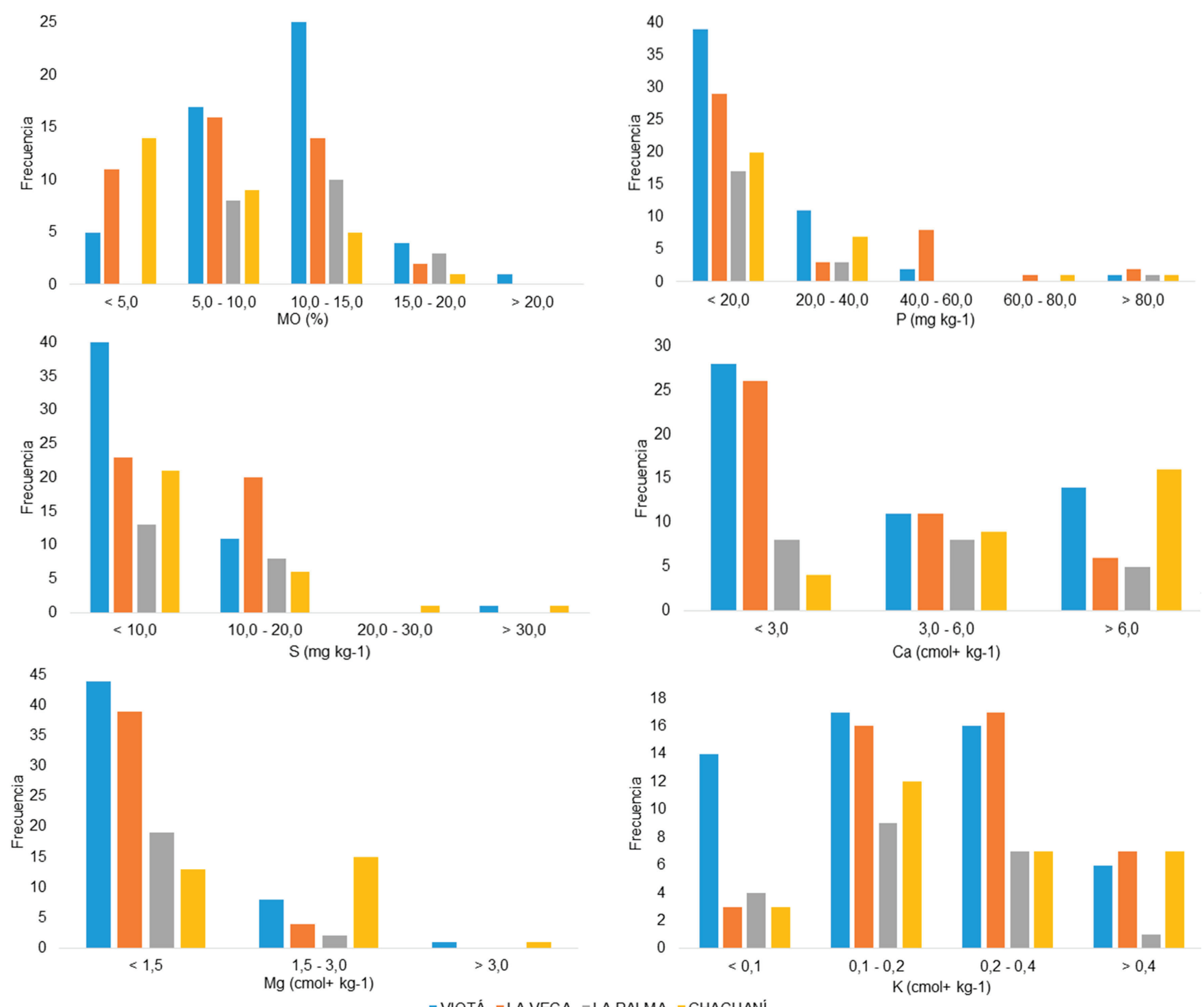

Figura 2. Análisis de frecuencias para las propiedades del suelo (MO, P, S, Ca, Mg y K), según una muestra de 146 fincas cultivadas con plátano, en los municipios de Viotá, La Vega, La Palma y Chaguaní, en el departamento de Cundinamarca (Colombia). Los rangos corresponden a los reportados en la Quinta aproximación del ICA (1992).

arbusculares (HMA) y solubilizadores de $\mathrm{P}$ (hongos y bacterias), que estimulan el aumento de la exploración radical y la disponibilidad de P (Lozano et al. 2015).

Los niveles de $\mathrm{S}$ en los suelos de los cuatro municipios fueron bajos, al presentar valores menores a $10 \mathrm{mg} \mathrm{kg}^{-1}$ (Figura 2) y mostraron correlación positiva con la $\mathrm{CE}(\mathrm{r}=0,61, \mathrm{P}<0,01)$, resultado que concuerda con lo encontrado por Sierra et al. (2007). Estos autores atribuyen esta relación al incremento de la $\mathrm{CE}$, debido a la reacción del $\mathrm{H}_{2} \mathrm{SO}_{4}$ con el $\mathrm{CaCO}_{3}$, que genera $\mathrm{CaSO}_{4}$, así como un aumento de aniones y cationes en el suelo. A pesar de esto, los niveles de salinidad encontrados en el suelo de las fincas son bajos y, por ende, no poseen niveles críticos que requieran acciones correctivas o representen niveles tóxicos para las plantas de plátano. Adicionalmente, se registró correlación positiva entre el S y el $\mathrm{K}(\mathrm{r}=0,65, \mathrm{P}<0,01)$, posiblemente, relacionada con la fertilización potásica por parte de los agricultores a las plantas de café, asociadas al cultivo de plátano, fundamentada en sulfato de potasio.

Para el caso de las bases, el Ca se encuentra en niveles medios a bajos $\left(<3 \mathrm{cmol}^{+} \mathrm{kg}^{-1}\right)$, en la mayoría (92) de los suelos de las fincas de los municipios de La Vega, Viotá y La Palma, mientras que para Chaguaní, se encontraron niveles altos $\left(>6 \mathrm{cmol}^{+} \mathrm{kg}^{-1}\right)$ (Figura 2). El $\mathrm{Mg}$, se encuentra en concentraciones bajas $\left(<1,5 \mathrm{cmol}^{+} \mathrm{kg}^{-1}\right)$; por su parte, el K está en niveles medios $\left(0,1-0,4 \mathrm{cmol}^{+} \mathrm{kg}^{-1}\right)$ en los suelos de los cuatro municipios (Figura 2). Adicionalmente, se encontraron desbalances entre los cationes, desfavoreciendo la absorción de $\mathrm{K}$ por parte de las plantas, lo cual, se relaciona con los pHs ácidos 
encontrados (Tabla 1) y la capacidad de extracción K del plátano. Teniendo en cuenta los resultados anteriores y observaciones en campo durante los muestreos, los niveles altos de Ca en los suelos de Chaguaní pueden estar asociados a malas prácticas de manejo, por la adición de enmiendas edáficas, tanto a los cultivos de café como a los de plátano.
Variabilidad de los suelos de los municipios productores de plátano. Según el análisis descriptivo de los resultados de los cuatro municipios del departamento de Cundinamarca, se presentaron diferencias significativas $(\mathrm{P}<0,05)$ en las propiedades $\mathrm{MO}$, CICE, $\mathrm{Na}, \mathrm{Ca}, \mathrm{Mg}, \mathrm{Fe}, \mathrm{Mn}, \mathrm{Zn}, \mathrm{B}$ y las relaciones catiónicas $\mathrm{Ca} / \mathrm{K}, \mathrm{Mg} / \mathrm{K}$ y $(\mathrm{Ca}+\mathrm{Mg}) / \mathrm{K}$ (Tabla 1). Cabe resaltar que los suelos de Chaguaní no

Tabla 1. Propiedades químicas de suelos (promedios y error estándar ( \pm )) cultivados con plátano en los municipios de Viotá, La Vega, La Palma y Chaguaní, en el departamento de Cundinamarca (Colombia), interpretación del promedio y resultados de la comparación entre los cuatro municipios, según análisis de varianza.

\begin{tabular}{|c|c|c|c|c|c|c|c|c|c|}
\hline \multirow[b]{2}{*}{ Municipio } & \multicolumn{7}{|c|}{ Promedios \pm error estándar / interpretación ${ }^{(2)}$} & & \multirow{2}{*}{$\begin{array}{l}\text { Anova }^{(1)} \\
\text { Valor } \mathbf{P}\end{array}$} \\
\hline & Viotá & & La Vega & & La Palma & & Chaguaní & & \\
\hline Observaciones & 53 & & 43 & & 21 & & 29 & & Ho: $\mu G=\mu S$ \\
\hline \multicolumn{10}{|l|}{ Propiedades } \\
\hline $\mathrm{pH}$ & $5,09 \pm 0,06$ & FA & $5,01 \pm 0,06$ & FA & $5,14 \pm 0,09$ & FA & $5,20 \pm 0,07$ & FA & 0,2551 \\
\hline MO (\%) & $10,53^{a} \pm 0,58$ & A & $8,35^{\mathrm{b}} \pm 0,64$ & A & $11,16^{\mathrm{a}} \pm 0,92$ & A & $6,26^{\mathrm{b}} \pm 0,78$ & A & 4,1040E-06* \\
\hline $\mathrm{CE}\left(\mathrm{dS} \mathrm{m}^{-1}\right)$ & $0,32 \pm 0,02$ & $\mathrm{~B}$ & $0,36 \pm 0,03$ & B & $0,34 \pm 0,04$ & B & $0,33 \pm 0,03$ & $\mathrm{~B}$ & 0,8009 \\
\hline $\mathrm{CICE}\left(\mathrm{cmol}^{+} \mathrm{kg}^{-1}\right)$ & $7,48^{\mathrm{b}} \pm 0,56$ & & $6,36^{\mathrm{b}} \pm 0,62$ & & $7,36^{\mathrm{b}} \pm 0,89$ & & $11,85^{a} \pm 0,76$ & & 2,8500E-08* \\
\hline $\mathrm{Al}+\mathrm{H}\left(\mathrm{cmol}^{+} \mathrm{kg}^{-1}\right)$ & $1,86 \pm 0,32$ & & $1,85 \pm 0,35$ & & $1,92 \pm 0,51$ & & $2,96 \pm 0,43$ & & 0,1587 \\
\hline $\mathrm{Na}\left(\mathrm{cmol}^{+} \mathrm{kg}^{-1}\right)$ & $0,13^{\mathrm{a}} \pm 0,03$ & B & $0,41^{\mathrm{a}} \pm 0,04$ & $\mathrm{~B}$ & $0,11^{\mathrm{ab}} \pm 0,05$ & B & $0,11^{\mathrm{b}} \pm 0,05$ & $\mathrm{~B}$ & 1,5040E-10* \\
\hline \multicolumn{10}{|l|}{$\begin{array}{l}\text { Elementos } \\
\text { mayores }\end{array}$} \\
\hline $\mathrm{P}\left(\mathrm{mg} \mathrm{kg}^{-1}\right)$ & $18,86 \pm 5,74$ & B & $34,75 \pm 6,38$ & M & $17,63 \pm 9,13$ & B & $19,23 \pm 7,77$ & B & 0,2141 \\
\hline $\mathrm{S}\left(\mathrm{mg} \mathrm{kg}^{-1}\right)$ & $8,05 \pm 0,72$ & B & $10,12 \pm 0,80$ & B & $9,07 \pm 1,15$ & B & $9,56 \pm 0,98$ & $\mathrm{~B}$ & 0,2730 \\
\hline $\mathrm{Ca}\left(\mathrm{cmol}^{+} \mathrm{kg}^{-1}\right)$ & $4,33^{\mathrm{b}} \pm 0,47$ & M & $3,41^{\mathrm{b}} \pm 0,52$ & M & $4,20^{\mathrm{b}} \pm 0,75$ & M & $6,92^{a} \pm 0,64$ & A & $0,0002 *$ \\
\hline $\mathrm{Mg}\left(\mathrm{cmol}^{+} \mathrm{kg}^{-1}\right)$ & $0,94^{\mathrm{b}} \pm 0,10$ & $\mathrm{~B}$ & $0,68^{\mathrm{b}} \pm 0,11$ & $\mathrm{~B}$ & $0,94^{\mathrm{b}} \pm 0,16$ & $\mathrm{~B}$ & $1,54^{\mathrm{a}} \pm 0,13$ & $\mathrm{M}$ & 5,4770E-06* \\
\hline $\mathrm{K}\left(\mathrm{cmol}^{+} \mathrm{kg}^{-1}\right)$ & $0,22 \pm 0,04$ & M & $0,30 \pm 0,04$ & M & $0,19 \pm 0,06$ & $\mathrm{~B}$ & $0,34 \pm 0,05$ & M & 0,1376 \\
\hline \multicolumn{10}{|l|}{$\begin{array}{l}\text { Elementos } \\
\text { menores }\end{array}$} \\
\hline $\mathrm{Fe}\left(\mathrm{mg} \mathrm{kg}^{-1}\right)$ & $264,14^{\mathrm{bc}} \pm 38,07$ & A & $415,80^{a} \pm 42,27$ & $\mathrm{~A}$ & $369,68^{\mathrm{ab}} \pm 60,49$ & $\mathrm{~A}$ & $187,55^{\mathrm{c}} \pm 51,47$ & $\mathrm{~A}$ & $0,0020^{*}$ \\
\hline $\mathrm{Cu}\left(\mathrm{mg} \mathrm{kg}^{-1}\right)$ & $2,34 \pm 0,58$ & M & $3,44 \pm 0,64$ & $\mathrm{~A}$ & $2,69 \pm 0,92$ & M & $1,77 \pm 0,78$ & M & 0,3898 \\
\hline $\operatorname{Mn}\left(\mathrm{mg} \mathrm{kg}^{-1}\right)$ & $2,92^{\mathrm{b}} \pm 0,49$ & $\mathrm{~B}$ & $4,47^{\mathrm{b}} \pm 0,54$ & $\mathrm{~B}$ & $3,54^{\mathrm{b}} \pm 0,77$ & $\mathrm{~B}$ & $6,28^{a} \pm 0,66$ & M & $0,0003^{*}$ \\
\hline $\mathrm{Zn}\left(\mathrm{mg} \mathrm{kg}^{-1}\right)$ & $3,62^{\mathrm{b}} \pm 1,05$ & $\mathrm{~A}$ & $9,53^{a} \pm 1,16$ & $\mathrm{~A}$ & $6,10^{\mathrm{b}} \pm 1,66$ & $\mathrm{~A}$ & $5,18^{\mathrm{b}} \pm 1,41$ & $\mathrm{~A}$ & $0,0016^{*}$ \\
\hline $\mathrm{B}\left(\mathrm{mg} \mathrm{kg}^{-1}\right)$ & $0,26^{\mathrm{a}} \pm 0,02$ & M & $0,29^{a} \pm 0,02$ & M & $0,17^{\mathrm{b}} \pm 0,03$ & $\mathrm{~B}$ & $0,20^{\mathrm{ab}} \pm 0,03$ & M & 0,0099* \\
\hline \multicolumn{10}{|l|}{$\begin{array}{l}\text { Relaciones } \\
\text { catiónicas }\end{array}$} \\
\hline $\mathrm{Ca} / \mathrm{Mg}$ & $4,47 \pm 0,19$ & A & $4,87 \pm 0,21$ & A & $4,61 \pm 0,29$ & A & $4,67 \pm 0,25$ & A & 0,5595 \\
\hline $\mathrm{Ca} / \mathrm{K}$ & $22,87^{\mathrm{b}} \pm 2,38$ & A & $13,80^{\mathrm{b}} \pm 2,65$ & $\mathrm{~A}$ & $24,34^{\mathrm{b}} \pm 3,79$ & A & $37,19^{a} \pm 3,22$ & A & 1,2400E-07* \\
\hline $\mathrm{Mg} / \mathrm{K}$ & $5,18^{\mathrm{a}} \pm 0,61$ & A & $2,87^{\mathrm{b}} \pm 0,67$ & $\mathrm{~B}$ & $6,05^{\mathrm{a}} \pm 0,96$ & A & $8,32^{a} \pm 0,82$ & A & 1,5820E-06* \\
\hline$(\mathrm{Ca}+\mathrm{Mg}) / \mathrm{K}$ & $28,07^{\mathrm{b}} \pm 2,94$ & $\mathrm{~A}$ & $16,59^{c} \pm 3,26$ & $\mathrm{~A}$ & $30,50^{\mathrm{ab}} \pm 4,67$ & A & $45,39^{a} \pm 3,97$ & $\mathrm{~A}$ & 1,1850E-07* \\
\hline
\end{tabular}

(1) Análisis de varianza.

(2) Interpretación, según la Quinta Aproximación del ICA: A alto, M medio y B bajo, FA fuertemente ácido.

(3) Superíndices demuestran las diferencias significativas entre municipios, por medio de la prueba de comparación múltiple de Tukey $(\mathrm{P}<0,05)$.

* Diferencias significativas al 5\% entre los promedios poblacionales $\mu$ de los cuatro municipios. 
presentaron valores promedios superiores respecto a los suelos de los demás municipios, en las variables CICE, Ca, Mg, Mn, mientras que los suelos de Viotá y La Palma presentaron valores superiores de MO; los de La Vega y Viotá, de Na y B y los de La Vega, de Zn y Fe.

Se realizó un análisis de componentes principales (ACP), en el que se incluyeron las variables que presentaron correlación, con al menos una. Por lo tanto, se excluyeron del análisis, el $\mathrm{Na}$ y los elementos menores $\mathrm{Cu}, \mathrm{Mn}$ y B, debido a su variabilidad independiente de las demás variables. El $\mathrm{Na}$ y el $\mathrm{Mn}$ presentaron niveles bajos y el $\mathrm{Cu}$ y B, niveles medios, en la mayoría de los suelos de las fincas (ICA, 1992); no obstante, el B es un elemento que asegura la estabilidad de las paredes celulares y, por tanto, hace parte fundamental en el proceso de formación del racimo, su deficiencia, promueve disminución en el peso y tamaño del fruto, así como reducción en el llenado de dedos (Taiz \& Zeiger, 2010). Por lo anterior, se deben realizar acciones correctivas, con el fin de evitar la deficiencia de micronutrientes. Las variables que entraron en el ACP fueron: $\mathrm{pH}, \mathrm{MO}, \mathrm{CE}, \mathrm{CICE}$, $\mathrm{Al}+\mathrm{H}, \mathrm{P}, \mathrm{S}, \mathrm{Ca}, \mathrm{Mg}, \mathrm{K}, \mathrm{Fe}$ y Zn. Se detectaron cuatro componentes que explican el $81,58 \%$ de la varianza del conjunto de las 12 variables (Tabla 2). Los componentes fueron extraídos por medio de la matriz de componente rotado, por el método de Varimax (Montoya Suárez, 2007).

Tabla 2. Aporte a la explicación de la varianza y vectores propios de los primeros cuatro factores del análisis de componentes principales sobre las propiedades del suelo cultivado con plátano, en los municipios de Viotá, La Vega, La Palma, Chaguaní, en Cundinamarca (Colombia).

\begin{tabular}{|l|c|c|c|c|}
\hline \multicolumn{1}{|c|}{ Aporte } & Factor 1 & Factor 2 & Factor 3 & Factor 4 \\
\hline \% de varianza & 27,42 & 19,92 & 19,06 & 15,17 \\
\hline Acumulado & 27,42 & 47,34 & 66,40 & 81,58 \\
\hline Vectores propios & 0,612 & $\mathbf{0 , 6 5 6}$ & $-0,134$ & 0,241 \\
\hline $\mathrm{pH}$ & $-0,113$ & $\mathbf{0 , 7 2 1}$ & 0,381 & $-0,193$ \\
\hline $\mathrm{MO}(\%)$ & 0,042 & 0,122 & $\mathbf{0 , 8 7 0}$ & 0,176 \\
\hline $\mathrm{CE}\left(\mathrm{dS} \mathrm{m}^{-1}\right)$ & $\mathbf{0 , 9 3 8}$ & $-0,213$ & 0,188 & 0,017 \\
\hline $\mathrm{CICE}\left(\mathrm{cmol}^{+} \mathrm{kg}^{-1}\right)$ & $-0,023$ & $\mathbf{- 0 , 8 5 9}$ & $-0,059$ & $-0,286$ \\
\hline $\mathrm{Al}+\mathrm{H}\left(\mathrm{cmol}^{+} \mathrm{kg}^{-1}\right)$ & 0,111 & $-0,012$ & 0,108 & $\mathbf{0 , 8 5 5}$ \\
\hline $\mathrm{P}\left(\mathrm{mg} \mathrm{kg}^{-1}\right)$ & 0,177 & 0,037 & $\mathbf{0 , 8 2 4}$ & 0,112 \\
\hline $\mathrm{S}\left(\mathrm{mg} \mathrm{kg}^{-1}\right)$ & $\mathbf{0 , 9 0 8}$ & 0,273 & 0,168 & 0,180 \\
\hline $\mathrm{Ca}\left(\mathrm{cmol}^{+} \mathrm{kg}^{-1}\right)$ & $\mathbf{0 , 9 0 3}$ & 0,194 & 0,191 & 0,094 \\
\hline $\mathrm{Mg}\left(\mathrm{cmol}^{+} \mathrm{kg}^{-1}\right)$ & 0,485 & 0,152 & $\mathbf{0 , 7 1 3}$ & 0,192 \\
\hline $\mathrm{K}\left(\mathrm{cmol}^{+} \mathrm{kg}^{-1}\right)$ & $-0,293$ & $\mathbf{- 0 , 7 1 1}$ & $-0,049$ & 0,262 \\
\hline $\mathrm{Fe}\left(\mathrm{mg} \mathrm{kg}^{-1}\right)$ & 0,130 & 0,004 & 0,253 & $\mathbf{0 , 8 5 0}$ \\
\hline $\mathrm{Zn}\left(\mathrm{mg} \mathrm{kg}^{-1}\right)$ & & & & \\
\hline
\end{tabular}

El primer componente está asociado a la capa de cambio del suelo y reúne las variables CICE, Ca y $\mathrm{Mg}$, presentado, a su vez, una alta correlación con la CICE $(\mathrm{r}=0,938, \mathrm{P}<0,01)$ (Tabla 2). Por otro lado, esta característica del suelo mostró interacción con las bases del complejo de cambio (Ca, Mg y K); sin embargo, no se presentó correlación con el K (Figura 3).

El segundo componente, se relaciona con la reacción del suelo y reúne las variables $\mathrm{pH}, \mathrm{MO}, \mathrm{Al}+\mathrm{H}$ y $\mathrm{Fe}$ (Tabla 2). La correlación entre el componente y la $\mathrm{Al}+\mathrm{H}$ fue alta y negativa $(\mathrm{r}=-0,859, \mathrm{P}<0,01)$, así como para el $\mathrm{Fe}(\mathrm{r}=-0,711, \mathrm{P}<0,01)$, mientras que para el $\mathrm{pH}$ y la MO fue alto y positivo $(\mathrm{r}=0,656, \mathrm{P}<0,01 ; \mathrm{r}=0,721, \mathrm{P}<0,01$, respectivamente). Estas características, se asocian con la disponibilidad de nutrientes en la solución del suelo y vislumbran la importancia de las fuentes de acidez en la capacidad de reacción del mismo.

Por otro lado, el tercer componente, se asocia con la salinidad del suelo y reúne las variables de CE, S y K (Tabla 2). La correlación obtenida con la CE fue positiva ( $\mathrm{r}=0,870, \mathrm{P}<0,01)$; aun así, los niveles de CE encontrados en las fincas son bajos $\left(<0,4 \mathrm{dS} \mathrm{m}^{-1}\right) \mathrm{y}$, por lo tanto, no representan un riesgo para las plantas de plátano. Finalmente, el cuarto componente reúne las variables P y Zn (Tabla 2), presentando una correlación significativa y positiva, tanto para el $\mathrm{P}$ como para el $\mathrm{Zn}(\mathrm{r}=0,855, \mathrm{P}<0,01 ; \mathrm{r}=0,850, \mathrm{P}<0,01$, respectivamente).

Cabe resaltar que el componente asociado a la capa de cambio del suelo mostró independencia frente al relacionado con la reacción del suelo (Figura 4). En este contexto, los suelos de La Vega y La Palma no difieren, debido a una tendencia ceñida a la izquierda y arriba, en la figura 4. Caso contrario a lo observado en los suelos de los municipios de Viotá y Chaguaní, que presentan diferencias en los componentes asociados a la reacción del suelo y la capa de cambio. Según lo anterior, a pesar de la tendencia mostrada por el $\mathrm{pH}$ en los suelos de los cuatro municipios, no tuvieron cambios significativos en la CICE. Aun así, el análisis de correlación simple 


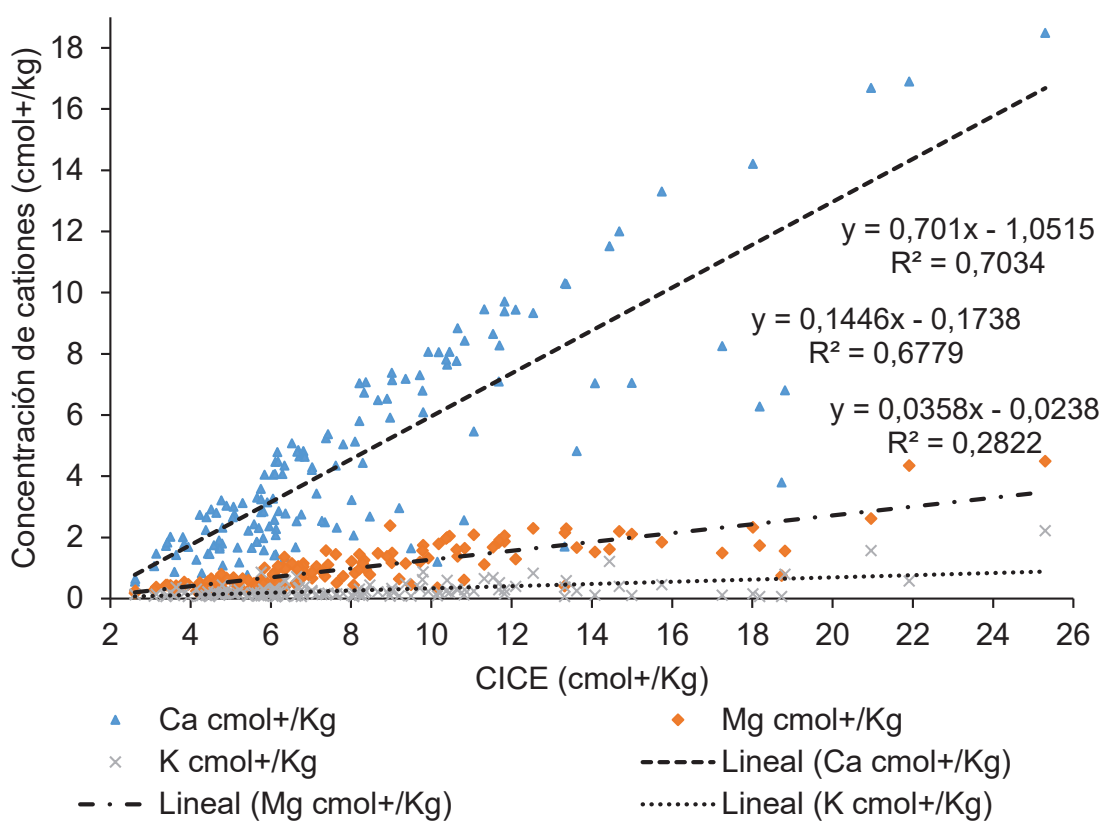

Figura 3. Interacciones entre la capacidad de intercambio catiónico efectivo (CICE) y los contenidos de cationes Ca y Mg, en suelos cultivados con plátano, en los municipios de Viotá, La Vega, La Palma y Chaguaní, en el departamento de Cundinamarca (Colombia).

por municipio mostró que el comportamiento entre los dos primeros componentes fue significativo para los municipios de Chaguaní y Viotá, positivo en el primer caso $(r=0,303, P=0,05)$ y negativo en el segundo $(\mathrm{r}=-0,442, \mathrm{P}<0,01)$, mientras que, para los suelos de los municipios de La Vega y La Palma, la correlación de estas dos características edáficas no fue significativa $(\mathrm{r}=0,173, \mathrm{P}=0,134 ; \mathrm{r}=$ $0,208, \mathrm{P}=0,183$, respectivamente).

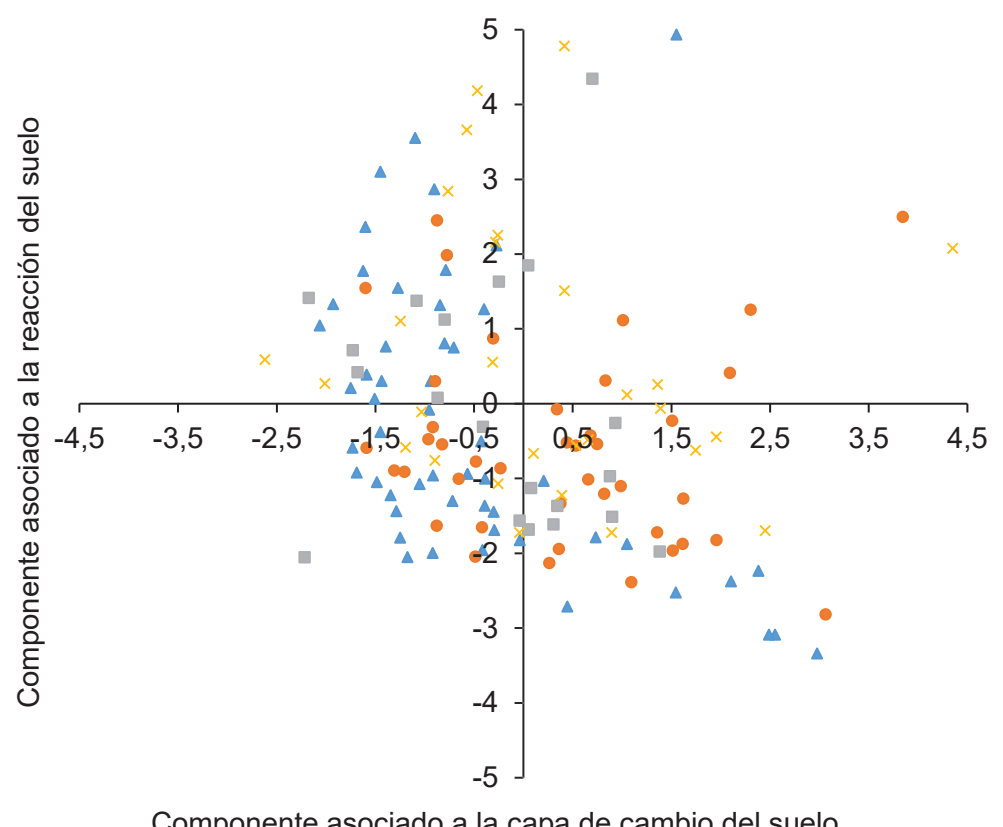

Componente asociado a la capa de cambio del suelo

$$
\text { ¿ Viotá - La Vega } \quad \text { La Palma } \times \text { Chaguaní }
$$

Figura 4. Comparación entre los componentes asociados a la reacción del suelo y propiedades de la capa de cambio de suelos cultivados con plátano en los municipios de Viotá, La Vega, La Palma y Chaguaní, en el departamento de Cundinamarca (Colombia). 
De acuerdo con el estudio de suelos realizado por el IGAC (2000), los suelos de La Vega y La Palma son Entisoles e Inseptisoles que, en su mayoría, están compuestos mineralógicamente por cloritas, vermiculitas parcialmente cloritizadas, illitas, entre otras. En este sentido, posiblemente la CIC de los suelos de estos dos municipios, se puede atribuir al tipo de arcillas predominantes en los perfiles, más que a la reacción del suelo (Otero et al. 1998). Cabe resaltar que la CICE y el contenido de cationes son el factor más determinante en la fertilidad de los suelos de los cuatro municipios, pero es importante realizar medidas correctivas, en cuanto al desbalance catiónico existente en la solución del suelo, ya que los niveles de $\mathrm{K}$ y Mg son muy bajos con relación a los de Ca. Es posible que los niveles bajos de K sean a causa de los niveles de extracción de las plantas de plátano, ya que requieren de altas cantidades de este elemento (Belalcázar, 1991), mientras que los niveles bajos de $\mathrm{Mg}$ y la disminución de los contenidos de Ca pueden estar asociados con procesos de lixiviación, que promueven la disminución del pH de los suelos (Meléndez \& Molina, 2001); lo anterior, junto a un manejo inadecuado de la fertilidad del suelo cada ciclo de cultivo, sugieren que se deben realizar aportes de estos elementos al suelo, tanto para aumentar su disponibilidad como para evitar desbalances catiónicos, que impidan su absorción por parte de las plantas y así lograr suplir sus necesidades.

El componente asociado con la reacción del suelo también es importante para determinar la disponibilidad de nutrientes para las plantas. Como se mencionó anteriormente, existe una correlación fuerte entre los contenidos de $\mathrm{MO}$ y el Fe presente en la solución del suelo, del mismo modo, entre el pH y la acidez intercambiable. Por tal razón, se infiere que el componente explica la disminución del pH con relación al aumento de la acidez intercambiable, además de la reducción de los contenidos de Fe, al incrementarse las cantidades de MO. Acorde con esto, la reacción de los suelos de las fincas muestreadas en el estudio puede depender de la cantidad de $\mathrm{MO}$ presente, ya que tiene la capacidad de acomplejar el Fe y Al, logrando ejercer control sobre la disponibilidad de estos elementos en la solución del suelo, disminuyendo la acidez intercambiable (Acevedo-Sandoval et al. 2004).

El componente tres, asociado a la salinidad de los suelos, a pesar de representar un porcentaje considerable de variabilidad, los niveles de $\mathrm{CE}$ en las fincas estudiadas, no afectan negativamente la disponibilidad de los nutrientes, ni el crecimiento y desarrollo de los cultivos de plátano. Por otro lado, los resultados observados en el componente cuatro sugieren que los altos contenidos de $\mathrm{Zn}$ en el suelo, se pueden haber favorecido por su reacción ácida (Fischer et al. 2012), influenciada, a su vez, por aplicaciones de plaguicidas y fertilizantes, que contienen el microelemento, lo cual, puede favorecer su acumulación en el suelo (Sparks, 2005). En cuanto al P, su contenido se puede ver limitado, debido a que forma pares iónicos con el Zn, estimulando la formación de fosfatos de Zn e influyendo a que su precipitación con los óxidos de $\mathrm{Fe}$ y $\mathrm{Al}$, no sea la única razón para disminuir la concentración de $\mathrm{P}$ en el suelo (Zhao et al. 1998; Acevedo-Sandoval et al. 2004).
Como conclusión, los suelos de las fincas cultivadas con plátano en los cuatro municipios de Cundinamarca presentan deficiencias en los contenidos de nutrientes, además de desbalances catiónicos, lo cual, puede influir en una baja absorción de nutrientes, por parte de las plantas.

El pH de los suelos resultó ser fuertemente ácido en la mayoría de las fincas estudiadas; adicionalmente, esta característica se correlaciona con los contenidos de MO. Se debe tener en cuenta que una mayor cantidad de materiales orgánicos en el suelo disminuirá la presencia de formas solubles de $\mathrm{Fe}$ y Al, por lo tanto, se recomienda la aplicación de MO estabilizada, con el fin de realizar una disminución de los contenidos de estos dos elementos en la solución del suelo, ya que ambos se encuentran presentes en niveles altos. En este sentido, la aplicación de materiales de origen orgánico al suelo podría conllevar a un aumento en el $\mathrm{pH}$, mayor disponibilidad de nutrientes e incremento en la población de organismos benéficos en el suelo.

Los suelos analizados en este estudio difieren significativamente en sus propiedades, aun así, en los municipios de La Vega y La Palma, no presentan mayor variación en cuanto a la capa de cambio y la reacción del suelo, caso opuesto observado en los municipios de Viotá y Chaguaní.

Los contenidos de $\mathrm{Mg}$ y K, en los suelos de los cuatro municipios, son bajos, por tal razón, se recomienda realizar aplicación de productos que suplan las necesidades de las plantas de plátano. Adicionalmente, se recomienda tener precaución con las aplicaciones de productos fitosanitarios que contengan altos contenidos de $\mathrm{Zn}$, ya que pueden causar fitotoxicidad (Chaney, 1993).

Se debe considerar realizar la aplicación de productos biológicos que contengan microorganismos capaces de realizar interacciones sinérgicas con las plantas y, con esto, contribuir al aumento de la capacidad de exploración de las raíces, la solubilización de nutrientes y su disponibilidad en el suelo.

Se recomienda realizar un estudio específico de las características edafoclimáticas en la zona productora de plátano del departamento de Cundinamarca, con el fin de plantear manejos adecuados de la fertilidad, en función de la extracción del cultivo, los productos disponibles en el mercado, la capacidad adquisitiva de los productores y la relación costo-beneficio generada y, así, garantizar la sostenibilidad de los sistemas de productivos.

Agradecimientos: La presente investigación se desarrolló en marco del Convenio Especial de Cooperación Derivado 2 Corredor Tecnológico Agroindustrial 2 CTA SCTeI No. 022 de 2013 y el subproyecto "Validación de estrategias tecnológicas disponibles para los cultivos de plátano y yuca mediante la implementación de la metodología PIPA en el departamento de Cundinamarca", suscrito entre la Corporación Colombiana de Investigación de Investigación Agropecuaria AGROSAVIA, la Universidad Nacional de Colombia, la Secretaría de Ciencia Tecnología e Innovación de la Gobernación de Cundinamarca y la Secretaría Distrital de Desarrollo Económico 
de Bogotá D.C. Por su colaboración, se agradece especialmente a agricultores, Alcaldías y Umatas de los municipios de Viotá, Chaguaní, La Vega y La Palma. Conflictos de intereses: El manuscrito fue preparado y revisado con la participación de todos los autores, quienes declaramos que no existe conflicto de intereses que ponga en riesgo la validez de los resultados presentados. Financiación: Este estudio fue financiado por la Gobernación de Cundinamarca y la Alcaldía Mayor de Bogotá, cuya ejecución estuvo a cargo de la Corporación Colombiana de Investigación Agropecuaria AGROSAVIA.

\section{REFERENCIAS}

1. ACEVEDO-SANDOVAL, O.; ORTÍZ-HERNÁNDEZ, E.; CRUZ-SÁNCHEZ, M.; CRUZ-CHÁVEZ, E. 2004. El papel de óxidos de hierro en suelos. Terra Latinoamericana. (México). 22(4):485-497.

2. BELALCÁZAR, S. 1991. El cultivo del plátano (Musa AAB Simmonds) en el trópico. Manual de asistencia técnica No 50. ICA-CIID-Comité Departamental de Cafeteros del Quindío-INIBAP-LAC. (Colombia). 376p.

3. CANTO, B.; OROZCO, M.; MARTÍNEZ, L.; MANZO, G.; JAMES, A.; RODRÍGUEZ, C.; ISLAS, I.; BELTRÁN, M.; GUZMÁN, S.; GARRIDO, E.; HIGUERA, I.; SANDOVAL, J. 2015. Hacia dónde va la ciencia en México. Ed. Conacyt/Academia mexicana de ciencia A.C/Consejo consultivo de ciencias. (México). 77p.

4. CASIERRA, F; AGUILAR, O. 2007. Estrés por aluminio en plantas: reacciones en el suelo, síntomas en vegetales y posibilidades de corrección. Una revisión. Rev. Col. Ciencias Hortícolas. (Colombia). 1(2):246-257. https:// doi.org/10.17584/rcch.2007v1i2.8701

5. CASTAÑEDA, D.; JARAMILLO, D.; COTES, D. 2014. Selección de propiedades del suelo espacialmente relacionadas con producción en el cultivo de banano. Ciencia del Suelo. (Argentina). 32(1):73-83.

6. CHANEY R.L. 1993. Zinc Phytotoxicity. In: Robson A.D. (eds) Zinc in Soils and Plants. Proceedings of the International Symposium on 'Zinc in Soils and Plants' held at The University of Western Australia, 27-28 September, 1993. (Australia). 150p. https://doi.org/10.1007/978-94011-0878-2

7. DEPARTAMENTO ADMINISTRATIVO NACIONAL DE ESTADÍSTICA- DANE. 2015. Encuesta Nacional Agropecuaria. Departamento Administrativo Nacional de Estadística. Colombia. Disponible desde internet en: https://www.dane.gov.co/index.php/estadisticas-portema/agropecuario/encuesta-nacional-agropecuaria-ena (con acceso el 23/08/2017).
8. ESPINOSA, J.; MOLINA, E. 1999. Acidez de suelo y encalado. International Plant Nutrition Institute-IPNI. (Costa Rica). 42p.

9. FISCHER, G.; PIEDRAHITA, W.; TORRES, W.; RUBIO, W.; LIGARRETO, G.; GUERRERO, E.; ORDUZ-RODRÍGUEZ, J.; MIRANDA, D. 2012. Manual para el cultivo de frutales en el trópico. Frutales Misceláneos. Produmedios. (Colombia).1020p.

10. HAVLIN, J.; BEATON, J.; TISDALE, S.; NELSON, W. 1999. Soil fertility and fertilizers: An introduction to nutrient management, 6th edition. Ed. Prentice Hall. (Estados Unidos de América). 503p.

11. INSTITUTO COLOMBIANO AGROPECUARIO, ICA.1992. Fertilización en diversos cultivos. Quinta aproximación. Manual de Asistencia Técnica No. 25. Instituto Colombiano Agropecuario. (Colombia). 64p.

12. INSTITUTO GEOGRÁFICO AGUSTÍN CODAZZI, IGAC. 2000. Estudio general de suelos y zonificación de tierras del departamento de Cundinamarca, primera edición. Instituto Geográfico Agustín Codazzi. Bogotá D.C. (Colombia). Tomo II. 284p.

13. KERVEN, G.; EDWARDS, D.; ASHER, C.; KOKO'T, S. 1989. Aluminium determination in soil solution. II. Short-term colorimetric procedures for the measurement of inorganic monomeric aluminium in the presence of organic acid ligands. Soil Research. (Australia). 27(1):91-102. https:// doi.org/10.1071/SR9890091

14. LESCOT, T. 2015. Close-up Banana: Genetic diversity of the banana. FruiTrop. (Francia). 231:98-102.

15. LOZANO, J.; ARMBRECHT, I.; MONTOYA, J. 2015. Hongos formadores de micorrizas arbusculares y su efecto sobre la estructura de los suelos en fincas con manejos agroecológicos e intensivos. Acta Agronómica. (Colombia). 64(4):289296. https://doi.org/10.15446/acag.v64n4.46045

16. MELÉNDEZ, G.; MOLINA, E. 2001. Fertilidad de suelos y manejo de la nutrición de cultivos en Costa Rica. Centro de investigaciones agronómicas, Universidad de Costa Rica. (Costa Rica). 144p.

17. MONTOYA SUÁREZ, O. 2007. Aplicación del análisis factorial a la investigación de mercados. Caso de Estudio. Universidad Tecnológica de Pereira. Scientia et Technica. (Colombia). 1(35):281-286. http://dx.doi. org/10.22517/23447214.5443

18. OTERO, L.; SASTRIQUEZ, F.; MORALES, M. 1998. Participación de la arcilla y la materia orgánica en la capacidad 
de intercambio catiónico de vertisoles de la provincia de Granma. Terra Latinoamérica. (México).16(3):189-194.

19. PAREDES-MENDOZA, M.; ESPINOZA-VICTORIA, D. 2010. Ácidos orgánicos producidos por rizobacterias que solubilizan fosfato: una revisión crítica. Terra Latinoamérica. (México). 28(1):61-70.

20. SADEGHIAN, S. 2016. La acidez del suelo una limitante común para la producción de café. Avances técnicos. Programa de investigación científica. Fondo nacional del café. Cenicafé. (Colombia). 12p.

21. SIERRA, C.; LANCELLOTI, A.; VIDAL, I. 2007. Azufre Elemental como Corrector del pH y la Fertilidad de Algunos Suelos de la III y IV Región de Chile. Agricultura Ténica. (Chile). 67(2):173-181.

22. SISTEMA DE INFORMACIÓN NACIONAL DE AGRICULTURA, GANADERÍA, ACUACULTURA Y PESCA, SINAGAP. 2015. Boletín situacional del plátano. Ministerio de Agricultura y Ganadería. Gran minga nacional agropecuaria. Coordinación general del sistema de información nacional. (Ecuador). 6p.
23. SPARKS, D. 2005. Toxic metals in the environment: the role of surfaces. Elements. (Estados Unidos de América). 1(4):193197. https://doi.org/ 10.2113/gselements.1.4.193

24. TAIZ, L.; ZEIGER, E. 2010. Plant Physiology. 5th Edition, Sinauer Associates Inc. (Inglaterra). 782p.

25. ZAPATA, R. 2004. Química de la acidez del suelo. Origen de la acidez en el suelo. Documento de trabajo. Universidad Nacional de Colombia, Sede Medellín. (Colombia).197p.

26. ZHAO, F.; SHEN, Z.; MCGRATH, S. 1998. Solubility of zinc and interactions between zinc and phosphorus in the hyperaccumulator Thlaspi caerulescens. Plant, Cell \& Environment. (Inglaterra). 21(1):108-114. https://doi. org/10.1046/j.1365-3040.1998.00270.x

27. ZOPPOLO, R.; FASIOLO, C. 2016. Análisis foliar en frutales: Herramienta de diagnóstico de alto retorno. Programa Nacional de Producción Frutícola. Revista INIA. (Uruguay). 47:27-28. 